\title{
CSPAD-140k - a Versatile Detector for LCLS Experiments
}

Sven Herrmann ${ }^{a}$, Sébastien Boutet ${ }^{b}$, Brian Duda ${ }^{a}$, David Fritz ${ }^{b}$, Gunther Haller $^{\mathrm{a}}$, Philip Hart ${ }^{\mathrm{a}}$, Ryan Herbst ${ }^{\mathrm{a}}$ Christopher Kenney ${ }^{a}$, Henrik Lemke ${ }^{b}$, Marc Messerschmidt ${ }^{b}$, Jack Pines ${ }^{a}$, Aymeric Robert ${ }^{b}$, Marcin Sikorski ${ }^{b}$, Garth Williams ${ }^{b}$

a SLAC National Accelerator Laboratory, Menlo Park, CA 94025, USA

${ }^{\mathrm{b}}$ LCLS, SLAC National Accelerator Laboratory, Menlo Park, CA 94025, USA

corresponding author: Sven Herrmann, herrmann@slac.stanford.edu, tel: +1 650 926-2983

SLAC National Accelerator Laboratory, 2575 Sand Hill Road, MS-94, Menlo Park, CA 94025, USA

potential referees:

Rainer Richter, rar@hll.mpg.de (MPI for physics, Munich, Germany)

Bernd Schmitt, bernd.schmitt@psi.ch, (Paul Scherrer Institut, Villigen, Switzerland)

Peter Siddons, siddons@bnl.gov, (Brookhaven National Lab, USA)

\begin{abstract}
With the successful operation of three 2.3 megapixel, $120 \mathrm{~Hz}$ readout rate, hybrid pixel array detectors at the Linac Coherent Light Source (LCLS), the SLAC National Accelerator Laboratory detector group is now exploring additional applications based on the same detector platform. These megapixel cameras are based on the Cornell-SLAC hybrid Pixel Array Detector (CSPAD).

The CSPAD platform is developed around the CSPAD ASIC, a 36 kilopixel device, each pixel at $110 \times 110 \mu \mathrm{m}^{2}$. Important characteristics of the CSPAD (room temperature operation, 14bit on chip digitization with a purely digital data interface, and scaling modularity) make it an effective choice for designing detector variants that are optimized for a range of experiments and applications.

One of the first spin-off detectors based on this proven CSPAD platform is the CSPAD-140k: a 140 kilopixel detector, with an active area of approximately $4 \times 4 \mathrm{~cm}^{2}$ and four ASICs, bundled in a small, inexpensive and easy-to-deploy package. Due to its versatility it has already been used successfully in several experiments at the CXI, XPP and XCS instruments at LCLS. The work also describes problems faced by scaling from a prototype system to a full size $x$-ray camera and presents the current status on the improvements achieved.
\end{abstract}

Keywords: hybrid pixel detector; free electron laser; electronics; ASIC; CSPAD;

\section{Introduction}

With the successful operation of three 2.3 megapixel, $120 \mathrm{~Hz}$ readout rate, hybrid pixel array detectors at the Linac Coherent Light Source (LCLS), the SLAC National Accelerator Laboratory detector group is now exploring additional applications based on the same detector platform. These megapixel cameras are based on the Cornell-SLAC hybrid Pixel Array Detector (CSPAD) [1][2][3], particularly the hybrid pixel detector ASIC. This ASIC, with $194 \times 185$ pixels of $110 \times 110 \mu m^{2}$ size, is the central component of the CSPAD platform. Two ASICs, bump bonded to a single $500 \mu \mathrm{m}$-thick high-resistivity silicon pixel array sensor, constitute a $2 \times 1$ module, which is the basic building block of all CSPAD cameras. The first detector variant based on this proven platform, the CSPAD-140k, uses two of these blocks, glued to a carrier, building up a $2 \times 2$ module with a total of about 140 kilopixels. The CSPAD-140k (Figure 1 ) is a 140 kilopixel detector, with an active area of approximately $4 \times 4 \mathrm{~cm}^{2}$, bundled in a small, low-cost and easy- 
to-deploy package, suitable for various synchrotron and FEL applications. Development and performance optimization of this detector will be discussed in the following paragraphs.

(Figure 1 here; caption: The CSPAD 140k detector, an evolution of the pixel array detectors used successfully with megapixel cameras at LCLS.)

\section{Electronics and ASIC}

The pixel architecture is shown in Figure 2. It consists of a charge sensitive amplifier with two gain settings for collecting the detected signal. The sample and hold stage captures the amplifier output voltage and stores it for digitization. A comparator triggers when an externally supplied ramp falls below the stored signal voltage. A counter counts the clock cycles from the ramp start to the time point when the comparator fires. This implements a single slope analog to digital (A/D) converter. The counter values of all pixels are then readout one after the other.

(Figure 2 here; caption: Circuit diagram for a single CSPAD pixel. Functional elements in this circuit are described in the text.)

Basic parameters of the CSPAD ASIC are summarized in Table 1 and [1][2][3].

pixel size

ASIC size

frame rate

effective noise

single 8keV Photon SNR

maximum photon count
$110 \mu \mathrm{m} \times 110 \mu \mathrm{m}$

$185 \times 194$ pixels

$120 \mathrm{~Hz}$

300e- in HG; 1000 e- in LG

7 in HG; 2 in LG

350 in HG; 2700 in LG

[Table 1; caption: Basic physical and performance parameters of the CSPAD ASIC. (HG: high gain, LG: low gain mode)]

The first measurements performed at LCLS with a large 2.3 megapixel camera revealed that the single photon gain is smaller than expected from simulations and measurements with the prototype system. A comparison is shown in Figure 3. (Figure 3 here; caption: Comparison of a Monte Carlo simulated pixel histogram and a measured histogram. The Monte Carlo simulation used the CSPADs design parameters. The measurement was performed with an early 2.3 megapixel CSPAD version. The histogram shows the noise peak and the single $8 \mathrm{keV}$ photon peak. The measured gain for small signals is 4 times smaller than expected.) Further studies with these cameras showed complex crosstalk behavior between the pixels, partially due to on-chip and partially due to chip-PC-board-system interaction. The CSPAD-140k is especially suited for debugging these effects, as the $2 \times 2$ module with 4 ASICs is considerably simpler than the 2.3 megapixel cameras with 64 ASICs, while still using similar electronics and power cycling features. The electronics used in the CSPAD-140k are also much more easily accessible for probing or making modifications.

Figure 4 shows the measured ramp function with and without the ASIC. It can be seen that the firing of all the comparators within a short time modifies the external ramp signal. The modified ramp shows an undershoot, which accelerates the firing of comparators that have not yet reached their firing level. (Figure 4 here; caption: Screenshot of the ramp measured with and without the ASICs. The difference between the two ramp functions is shown in the lower trace with a fivefold magnification of the amplitude. The ramp exhibits undershoot when most of the comparators fire and the counters stop 
counting.) Another aspect of this inter-pixel crosstalk is the modification of the ASIC bias voltages. Figure 5 shows the four external bias nodes during the A/D conversion process. (Figure 5 here; caption: Screenshot of the ASIC bias nodes during the A/D conversion process. When most pixels stop counting the comparator bias nodes exhibit undershoot.) When most of the comparators fire, the bias voltage nodes of the comparators exhibit undershoot. This undershoot increases the effective bias current in the comparators, which in turn makes the comparators faster and therefore fire earlier, increasing the correlation of comparator firing. This effect is amplified by the on-board circuitry that is needed for power cycling the LCLS 2.3 megapixel CSPAD cameras: in order to switch the biases on and off at a rate of $120 \mathrm{~Hz}$, the nodes cannot simply be tied to a constant voltage. The local power regulators, which have to serve up to $16 \mathrm{ASICs}$, cannot respond fast enough to sudden changes in supply current when many counters stop clocking. In the current compact camera design, the impulse response on the power nodes feeds through to the ramp generation circuitry.

The small signal gain of a pixel depends on the actual ramp steepness the pixel sees around its pedestal value, and therefore on the comparator firing time. Figure 6 shows the measured variation of the gain of a pixel plotted against its average pedestal value (Figure 6 here; caption: Measured variation of the gain of a CSPAD pixel plotted against its average pedestal value. After the first pixel comparators fire the gain breaks down and most pixels show a small gain. Pixels with comparators which fire late show almost nominal gain). It can be seen that the majority of the pixels exhibit a smaller gain for small signals. This apparent smaller gain also affects the measured noise. In contrast, large signals will show the real gain. Furthermore, a camera showing the above effects can exhibit large signal crosstalk when areas of an ASIC are heavily illuminated, resulting in pedestal changes of the non-illuminated regions. As designed, the comparators in illuminated pixels fire later; therefore, their crosstalk contribution is missing at the time the comparators of the non-illuminated pixels fire. Due to this sequence, the comparators in the non-illuminated regions will fire on average a bit later, resulting in an unexpected increase of their digital pedestal value.

Modifications in the PCB power supply and improved bias and ramp generation have helped mitigate pixel crosstalk, and the new resulting cameras show a behavior much closer to the expected performance. As an example of the improved performance, Figure 7 shows a single photon histogram of copper fluorescence from a typical pixel measured with the CSPAD-140k. (Figure 7 here; caption: Measured histogram of a CSPAD-140k pixel illuminated with copper fluorescence in high gain mode.)

\section{CSPAD-140k: camera and applications at LCLS}

The pulsed nature of the LCLS allows using very short integration times. The CSPAD's hybrid pixel design enables electronic shuttering with signal integration times in the microsecond range; therefore, the detector can operate at room temperature without performance degradation from leakage current. For operation in vacuum, the temperature is stabilized by water cooling. For applications which need longer integration times, like experiments at synchrotrons, the design also supports utilization of a Peltier element to cool the detector. Figure 1 shows the basic elements of the CSPAD-140k camera. The camera housing, which includes the detector-ASIC hybrid and support electronics, is approximately $23.6 \mathrm{~cm}$ long, $4.4 \mathrm{~cm}$ wide and $4.8 \mathrm{~cm}$ tall. Behind a black kapton window is a $2 \times 2$ module with approximately $4 \mathrm{~cm} \mathrm{x}$ $4 \mathrm{~cm}$ active area and about 140 kilopixels. The CSPAD-140k module uses a fiber-optic interface to transfer data to the data acquisition system. Power and trigger signals are routed via a 26pin DSUB connector. The result is a small but robust interface which enables convenient deployment of the detector in an experiment chamber. 
A variant designed with slight modification to the package has been built for installation at the MEC instrument, where it will be used as a suitable alternate detector capable of $120 \mathrm{~Hz}$ readout, replacing a slow CCD camera in a Thomson spectrometer setup. Figure 8 shows a drawing of the MEC spectrometer with the CSPAD-140k (Figure 8; caption: View of a CSPAD-140k integrated as detector of a spectrometer in which will be used by the MEC instrument at LCLS).

A major advantage of the small and modular design of the CSPAD-140k is the capability to deploy multiple detectors in various mechanical arrangements. An interesting application of multiple CSPAD$140 \mathrm{k}$ modules is to tile them into an arc-like configuration in order to cover a larger angle relative to the interaction point. The multiple single-detector units represent a dedicated compound detector from the data acquisition system point of view. Figure 9 shows the concept for an upcoming experiment at the CXI instrument at LCLS (Figure 9; caption: View of an array of CSPAD-140k detectors organized in a compound detector; this design is under consideration for an upcoming experiment using the CXI instrument at LCLS).

The CSPAD-140k has already been used successfully in several experiments using the CXI [4], XPP and XCS instruments at LCLS. As an example, Figure 10 shows a single shot small angle scattering image recorded with CSPAD-140k using the XCS instrument. A dried colloidal dispersion was illuminated with unfocused monochromatic beam of $9.48 \mathrm{keV}$ energy. The detector was located $7.8 \mathrm{~m}$ behind the sample.

(Figure 10; caption: Single shot image recorded with the CSPAD-140k using the XCS instrument of LCLS. A dried colloidal dispersion is illuminated with the LCLS unfocused beam at 9.48keV energy. The detector was located $7.8 \mathrm{~m}$ behind the sample).

\section{Conclusions}

A small versatile $x$-ray camera based on the CSPAD platform has been built at SLAC. This system helped to characterize and improve the performance of all CSPAD cameras. In addition the CSPAD-140k has already been used for several experiments at LCLS and many more applications for this detector at synchrotrons and FELs are expected.

\section{Acknowledgements}

This work is supported by the Department of Energy, Laboratory Directed Research and Development funding, under contract DE-AC02-76SF00515. Portions of this research were carried out at the Linac Coherent Light Source, a National User Facility operated by Stanford University on behalf of the U.S. Department of Energy (DOE), Office of Basic Energy Sciences (OBES)

\section{References}

[1] L.J. Koerner, H.T. Philipp, M.S. Hromalik, M.W. Tate, S.M. Gruner, X-ray tests of a Pixel Array Detector for coherent x-ray imaging at the Linac Coherent Light Source, J Instrum, 4 (2009).

[2] H.T. Philipp, M. Hromalik, M. Tate, L. Koerner, S.M. Gruner, Pixel array detector for X-ray free electron laser experiments, Nucl Instrum Meth A, 649 (2011) 67-69.

[3] L.J. Koerner, M.S. Hromalik, M.W. Tate, S.M. Gruner, Femtosecond Radiation Experiment Detector for X-Ray Free-Electron Laser (XFEL) Coherent X-Ray Imaging, IEEE Transactions on Nuclear Science, 57 (2010) 5p. 
166 [4] R. Alonso-Mori et al, Shot-by-Shot Energy-Dispersive X-ray Emission Spectroscopy Using an X-ray Free 167 Electron Laser. Submitted for publication. 
Click here to download high resolution image

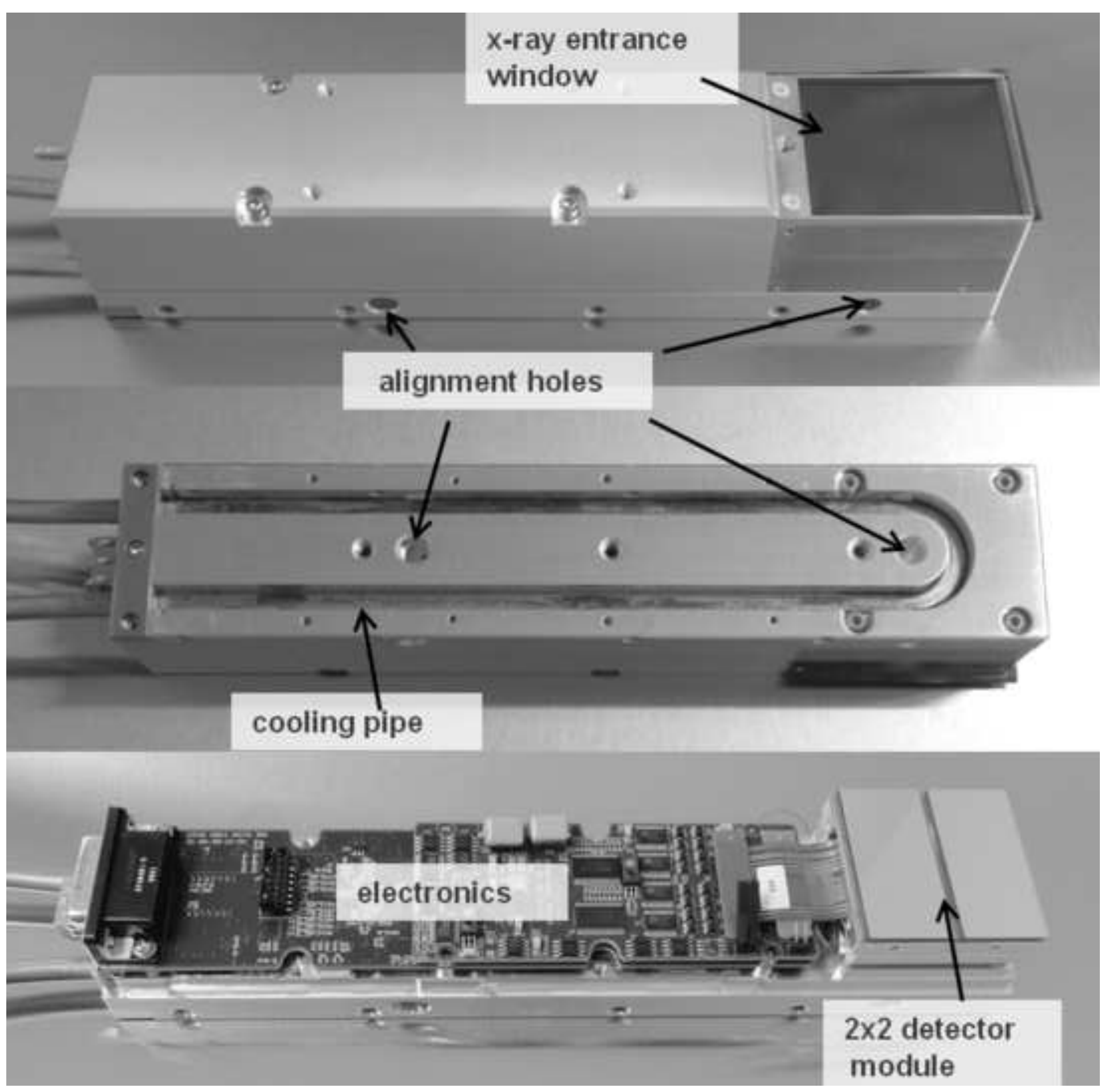




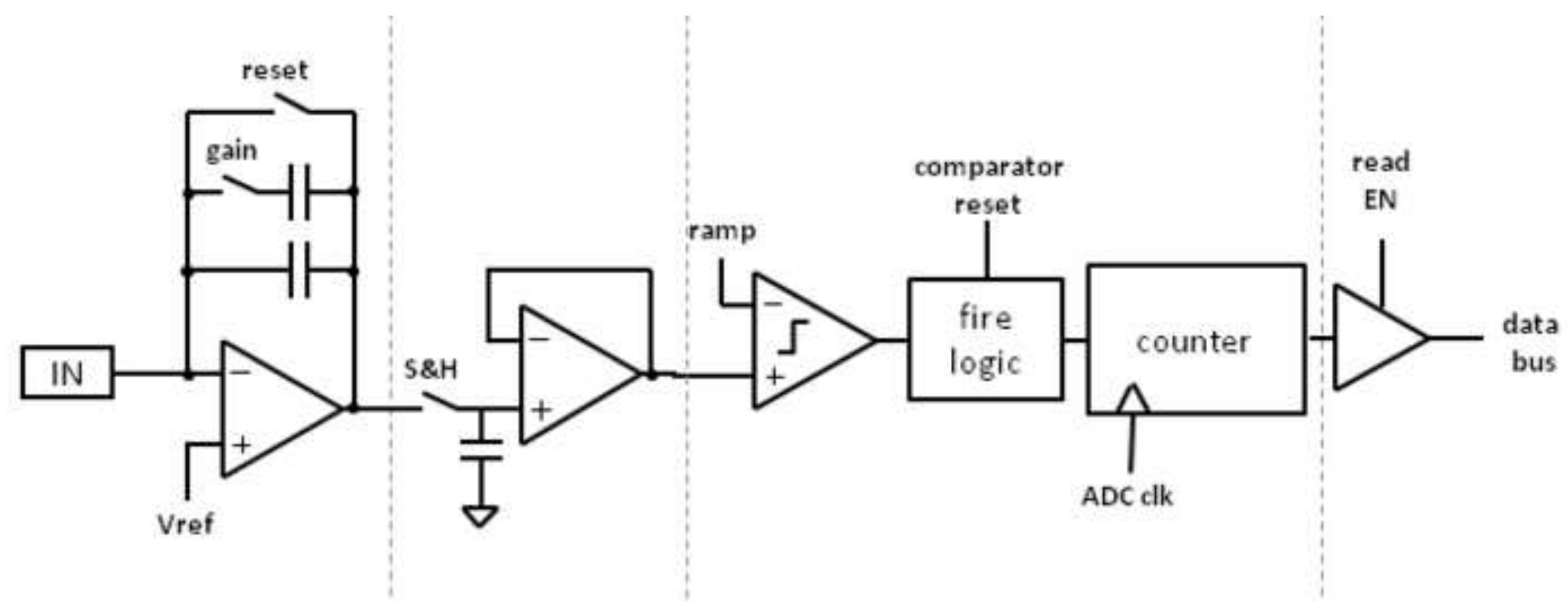




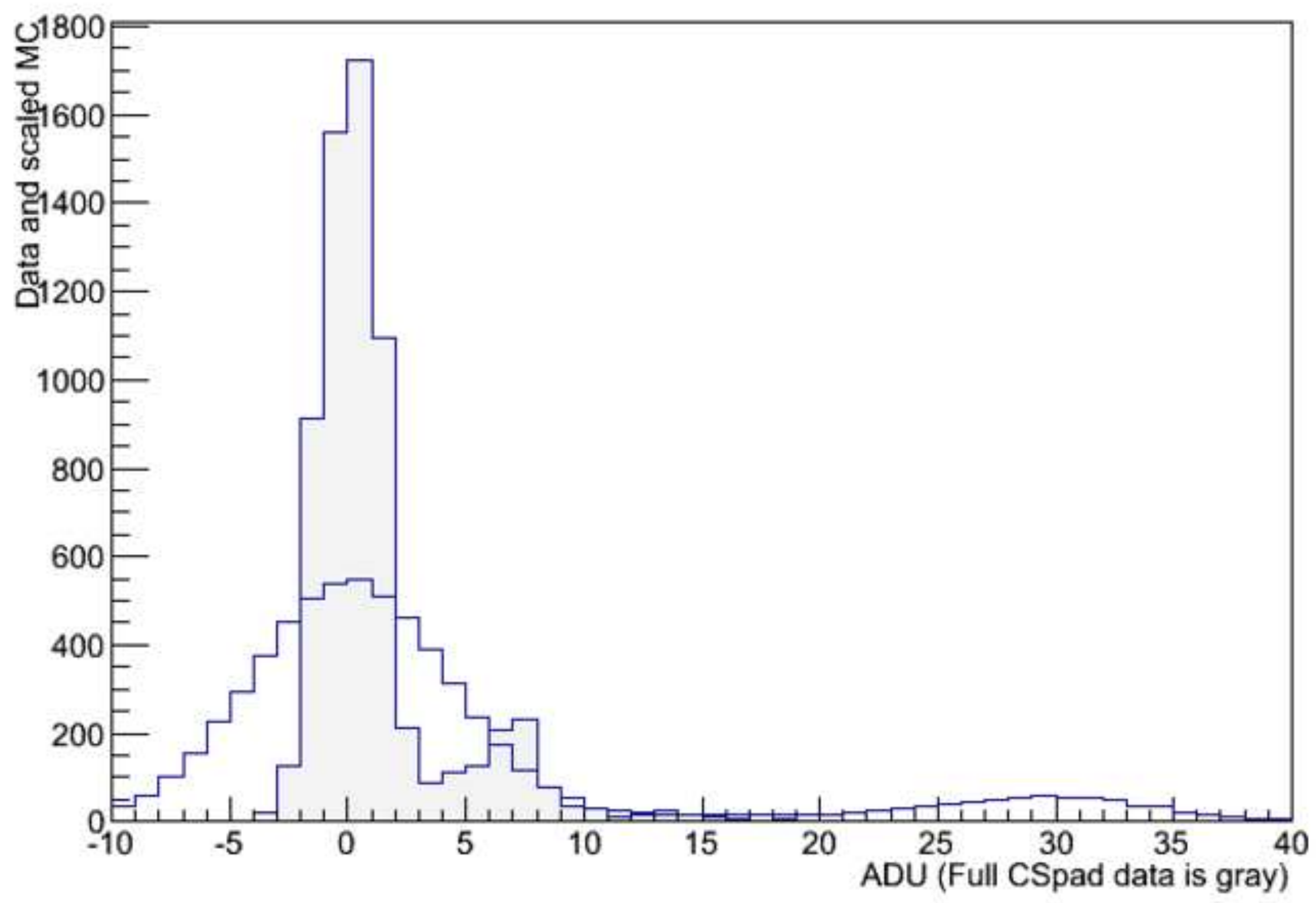


Click here to download high resolution image

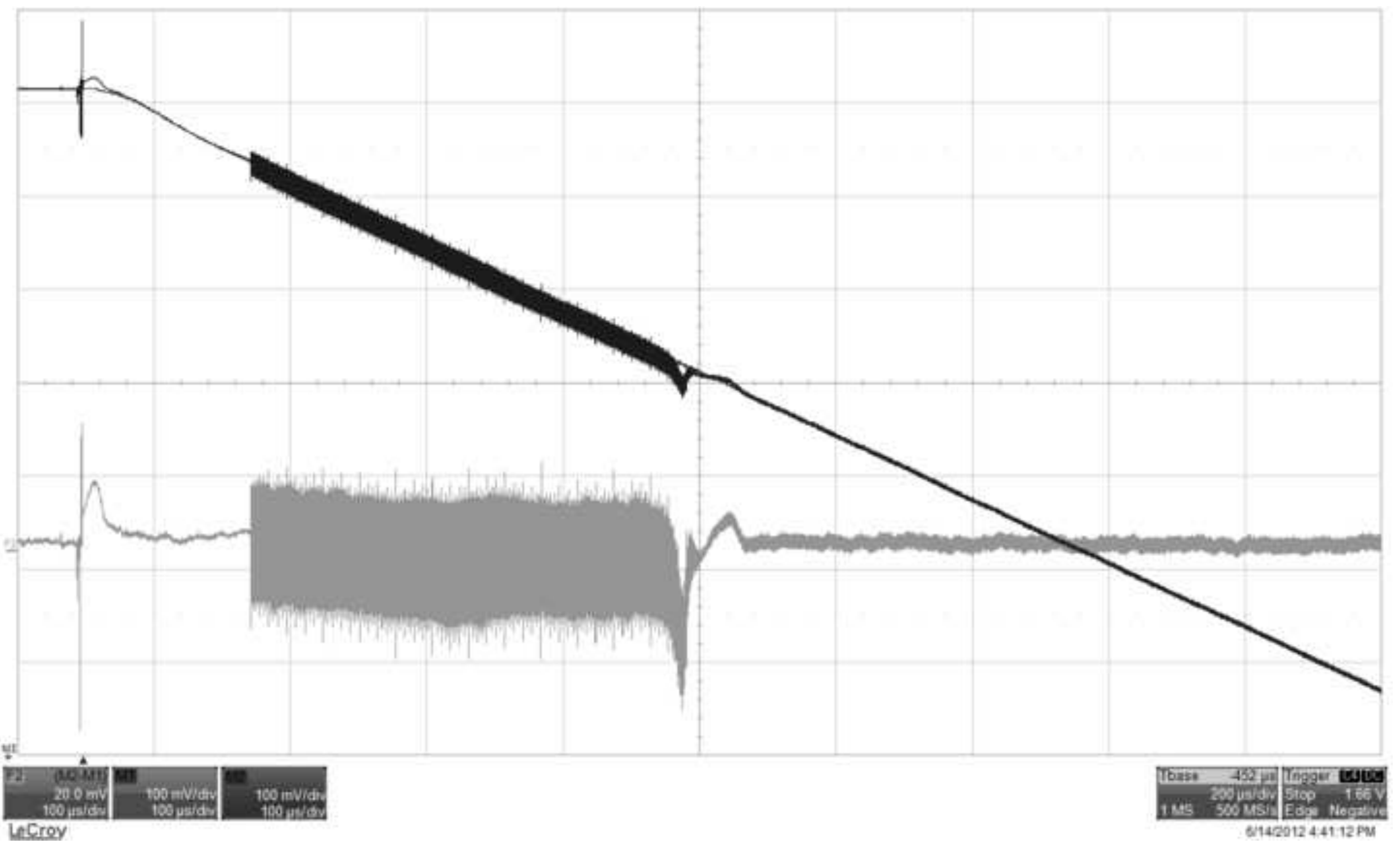


Figure 5
Click here to download high resolution image

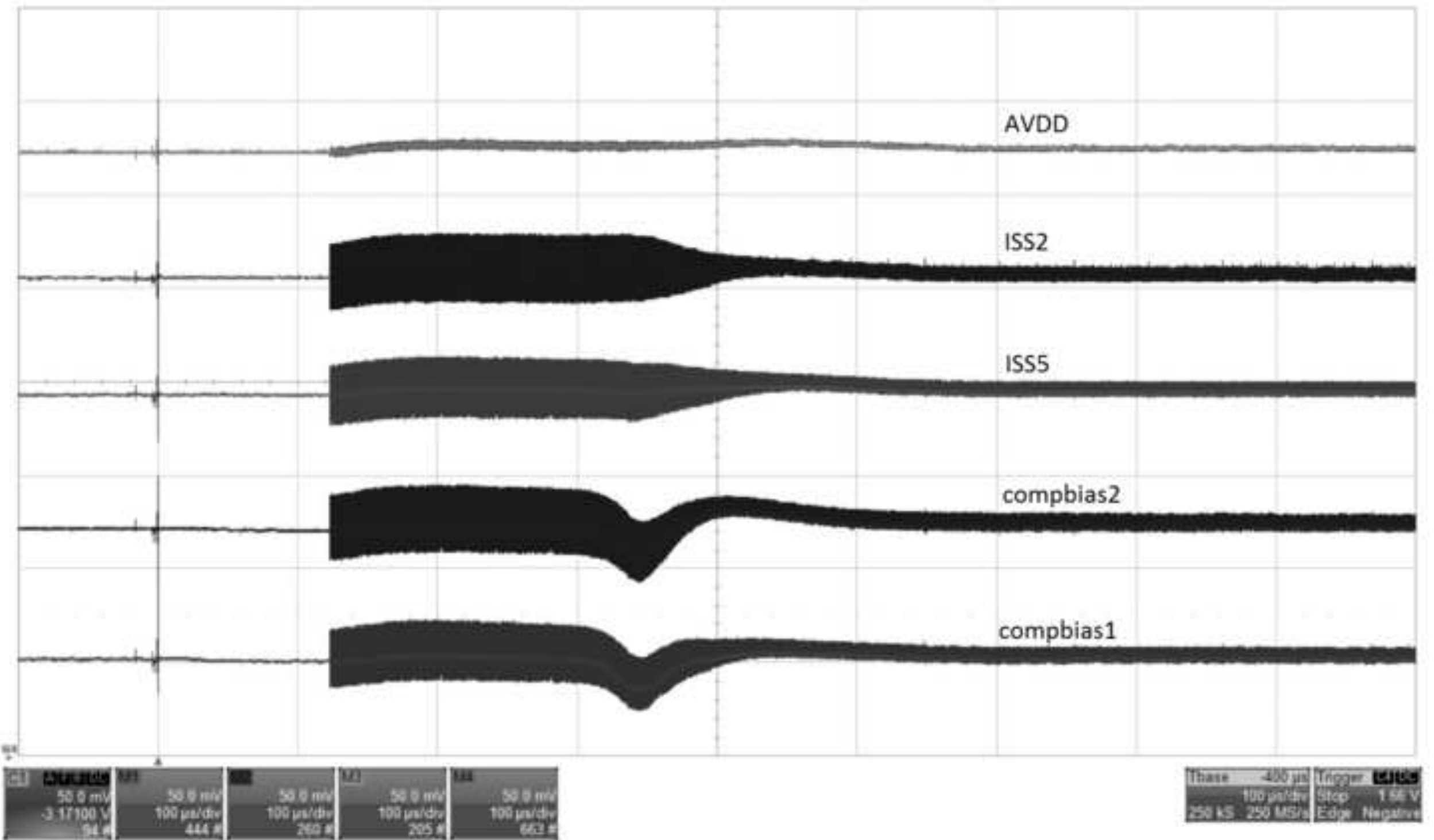




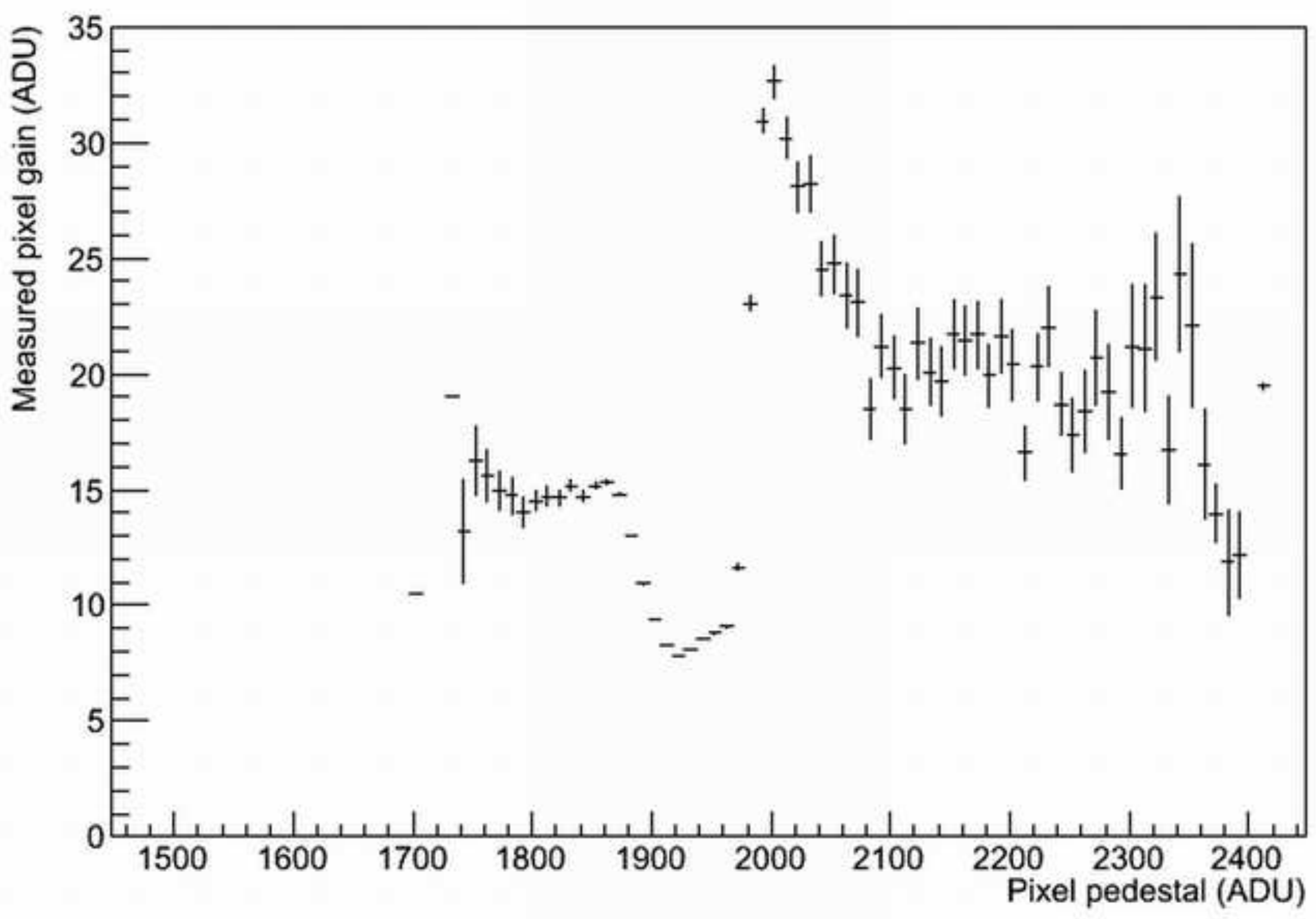




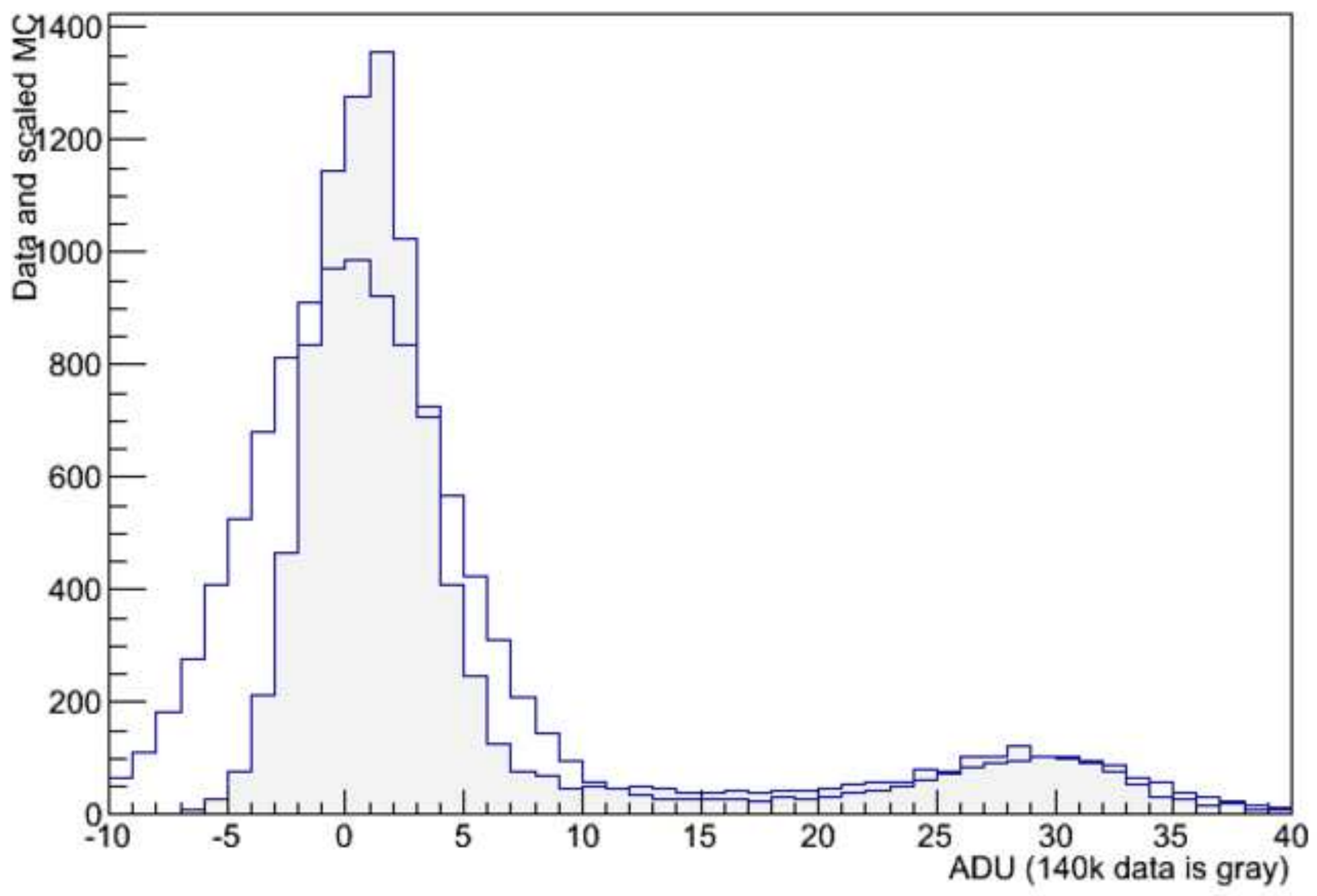


Click here to download high resolution image

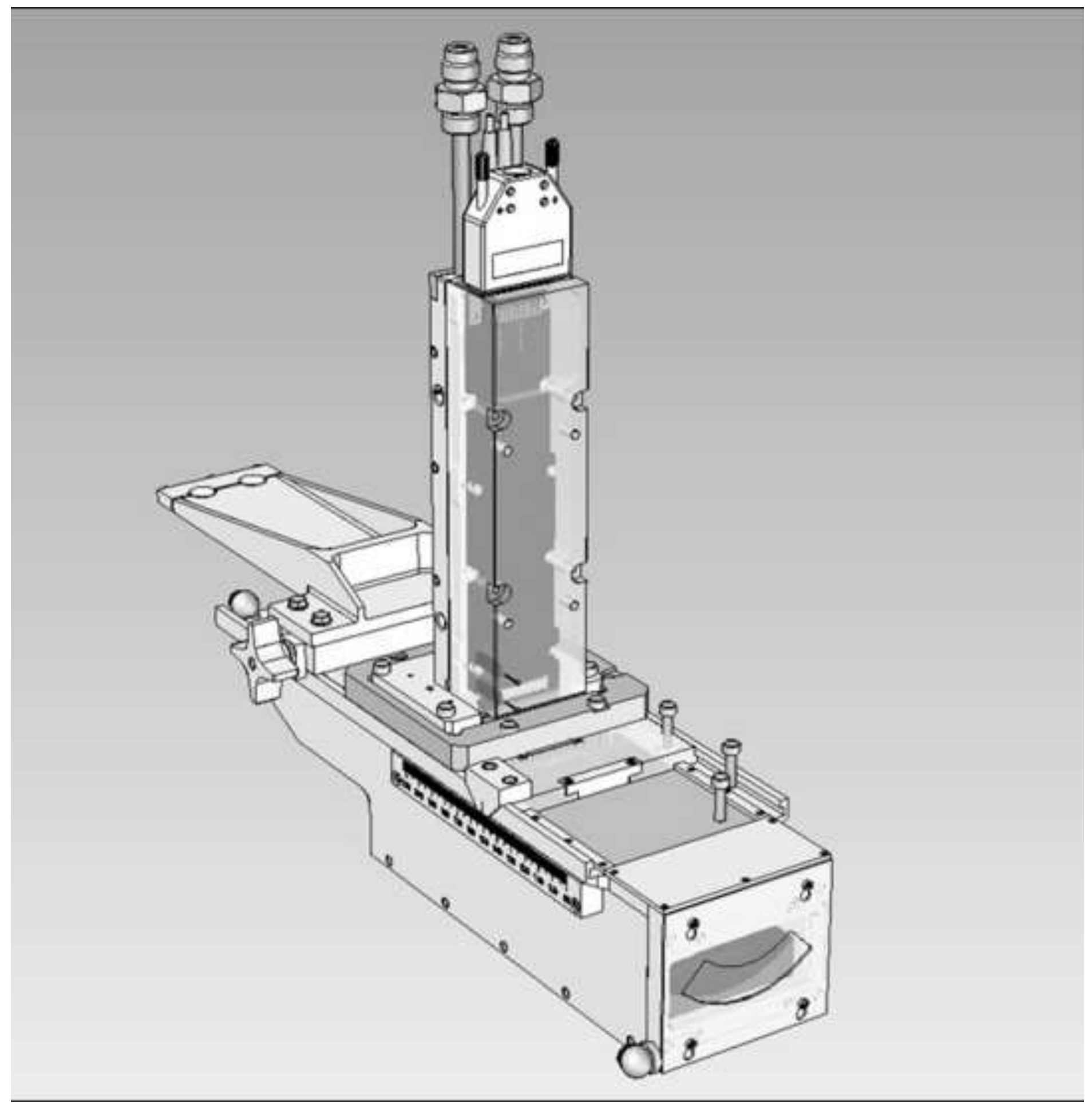




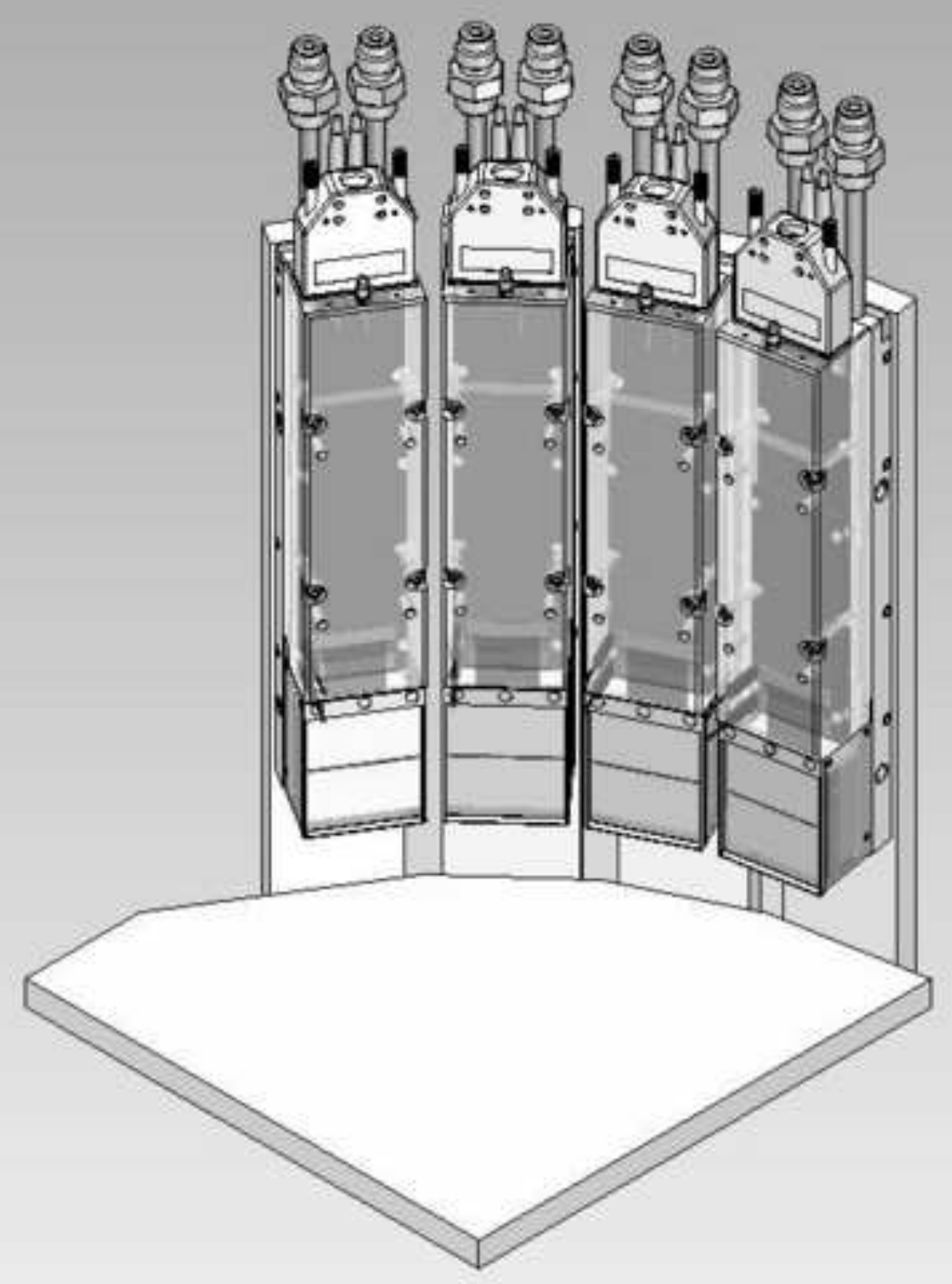


Figure 10 (color)
Click here to download high resolution image

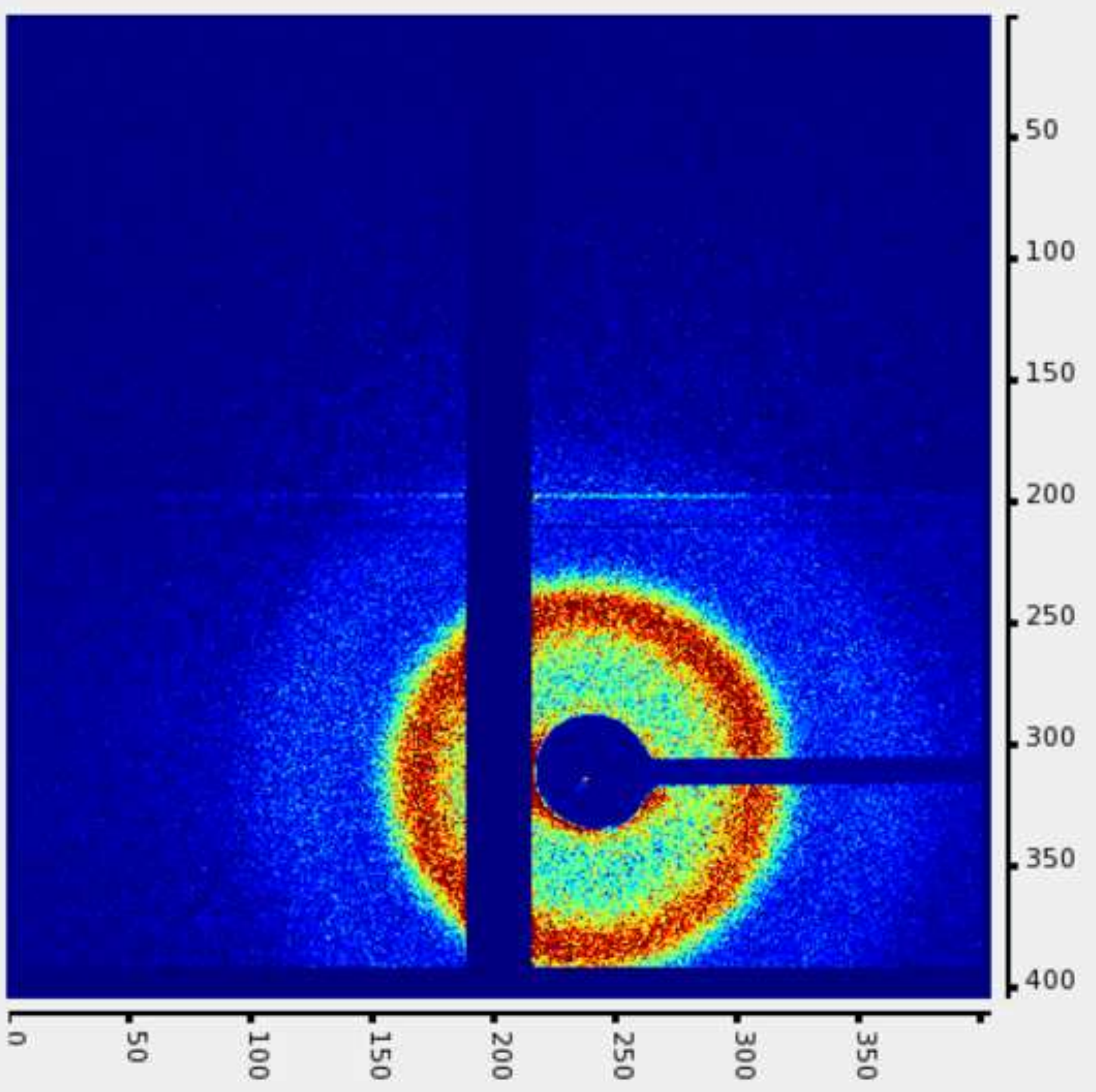



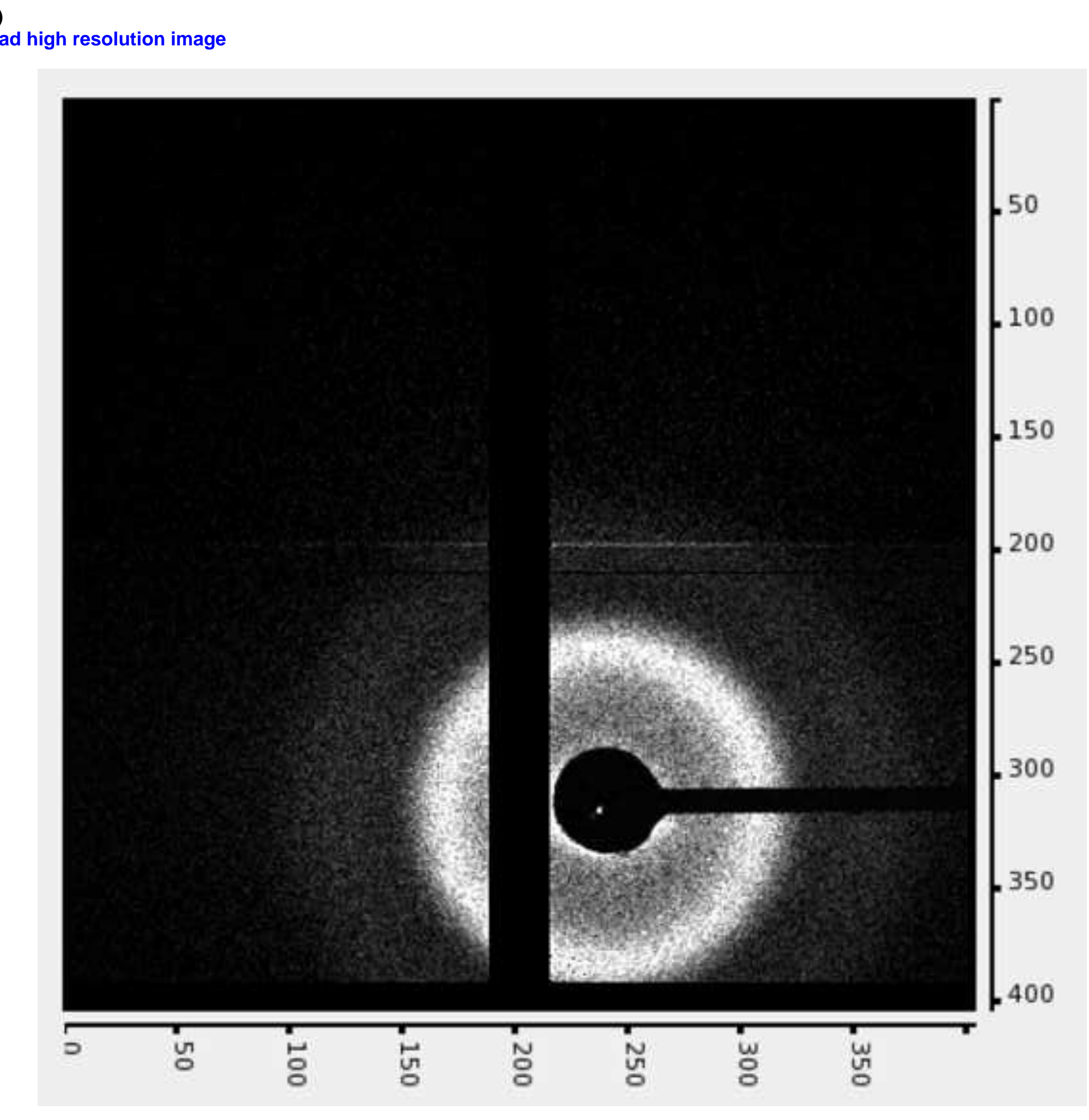

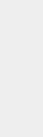

列

